\title{
Evaluación de las actitudes hacia la diversidad sexual de adolescentes del ámbito semi-rural gallego
}

\section{Assessment of attitudes towards sexual diversity of Galician adolescent's}

\section{semi-rural area}

\author{
Yolanda Rodríguez-Castro, Patricia Alonso-Ruido \\ Universidade de Vigo.
}

\begin{abstract}
Resumen
El objetivo de este estudio consiste en analizar las actitudes hacia la diversidad sexual de los y las adolescentes del ámbito semi-rural de la provincia de Ourense. Para ello contamos con una muestra de 150 adolescentes del $2^{\circ}$ ciclo de la E.S.O, con una media de edad de 15.9. Los resultados evidencian que los chicos son más homófobos que las chicas. Destacar que los chicos muestran actitudes más positivas hacia el lesbianismo que hacia la homosexualidad. Los chicos y las chicas más sexistas hostiles son los que presentan actitudes más negativas hacia el lesbianismo y la homosexualidad. También se ha detectado que los/as adolescentes del ámbito semi-rural presentan mayores niveles de homofobia y de sexismo.

Palabras clave: homofobia, lesbofobia, sexismo, adolescentes, semi-rural.
\end{abstract}

\begin{abstract}
The objective of this study is to evaluate the attitudes toward sexual diversity of adolescents from the semi-rural area of the province of Ourense. We have a sample of 150 adolescents from the 2 nd cycle of the ES, with an average age of 15.9. The results show that boys are more homophobic than girls. The boys show more positive attitudes toward lesbianism than toward homosexuality. The most hostile sexist boys and girls have more negative attitudes toward lesbianism and homosexuality. And adolescents in the semi-rural area have higher levels of homophobia and sexism.

Keywords: Homophobia, lesbophobia, sexism,
\end{abstract} adolescents, semi-rural.

Las actitudes hacia la diversidad sexual presentes en la sociedad actual están alimentadas por la ideología patriarcal que se sigue nutriendo de los estereotipos de género y la heteronormatividad (Lameiras, Carrera \& Rodríguez, 2013). El término homofobia fue acuñado a finales de la década de los 60 por George Weinberg, que lo definió como una actitud de miedo y rechazo hacia la homosexualidad. La homofobia implica un prejuicio, una actitud discriminatoria dirigida hacia una persona en función de su identidad homosexual que se caracteriza por un sentimiento de superioridad, que le lleva a la deshumanización del otro, y a la convicción de ser merecedor de privilegios (Viñuales, 2002). Una definición más abarcadora de homofobia sería el miedo o el pánico, de los hombres a amar a otros hombres, de hecho la identidad masculina tradicional se construye sobre la misoginia y la homofobia (Lameiras et al., 2013). Desde esta perspectiva, la homofobia no sólo afecta a los hombres homosexuales, sino a todas las personas que no se adaptan a los estereotipos tradicionales impuestos para su género con la amenaza de degradarles socialmente y ridiculizarlos (Guash, 2006).

La evolución del prejuicio hacia las personas homosexuales y lesbianas ha seguido un camino paralelo a los estudios del racismo y del sexismo (Aosved \& Long, 2006) que identificaron que los valores de racismo y de sexismo en las últimas décadas se han recanalizado hacia nuevas formas más sutiles de expresión que se han etiquetado como racismo ambivalente (Katz, Wackenhut \& Hass, 1986), racismo moderno (McConahay \& Hough, 1986; Pettigrew, 1989) y de forma similar, casi una década después, como sexismo moderno (Swim, Aikin, HaII \& Hunter, 1995), neosexismo (Tougas, Brown, Beaton \& Joly, 1995) y/o sexismo ambivalente (Glick \& Fiske, 1996). Así, las actitudes homófobas, abarcan desde las actitudes discriminatorias más hostiles y manifiestas hasta aquellas otras que, de una forma latente y sutil, permanecen silenciadas e invisibles (Quiles, Betancor, Rodríguez \& Rodríguez, 2003). En este sentido, podríamos afirmar que existen actitudes homófobas manifiestas y hostiles, tales como la agresión verbal, física o psicológica hacia las personas homosexuales; y actitudes homófobas latentes o sutiles, tales como los discursos culturales o médicos sobre el carácter patológico de la homosexualidad o la incapacitación de las parejas homosexuales para la adopción, entre otras cuestiones que pretenden coartar la vivencia y expresión homosexual.

La homofobia y el lesbianismo están fuertemente arraigadas en muchos países europeos, así lo demuestra el Informe del Consejo Europeo de 2011, ya que ambas han sido identificadas en los 47 estados miembros pertenecientes al Consejo de Europa, no obstante las 
actitudes varían significativamente entre los distintos países. Recientemente se ha publicado el informe Homofobia de Estado (ILGA, 2017) que afirma que a nivel mundial se ha avanzado en relación a los derechos LGTBI (Lesbianas, Gays, Transexuales, Bisexuales e Intersexuales) aunque no al ritmo esperado. En 121 Estados miembros de las Naciones Unidas ya no se imponen sanciones legales por la actividad sexual entre personas del mismo sexo, de manera privada y consensual. Pero siguen existiendo 73 Estados en los que se persiguen y criminalizan al colectivo LGTBI. Otro dato a destacar es que en más de 21 países, se reconoce el derecho a casarse de las parejas homosexuales/lesbianas. En dicho informe, también se presenta a nivel europeo un ranking de los países más respetuosos con los derechos del colectivo LGTBI. Sitúan a Malta en la cabeza del ranking, seguido por Noruega y Reino Unido. España que estuvo en la zona más alta, como lleva unos años sin avances, ya en el año 2016, se situó en quinto lugar y en el actual año, 2017, pasa a la novena posición. A la cola en derechos se sitúan Armenia, Rusia y Azerbaiyán.

En España, no existen demasiados estudios que se centren en el estudio de la homofobia/lesbofobia (España, Guerrero, Farré, Canella \& Abós, 2001; Martín Romero, 2008; Quiles et al., 2003), y específicamente en el colectivo de adolescentes (Pichardo, 2007; Injuve, 2008). Los resultados muestran que el $30 \%$ de los estudiantes ha participado en acciones homofóbicas como insultar; además también comprobaron que el 65\% de los chicos homosexuales han sufrido insultos, un $30 \%$ exclusión, un $20 \%$ golpes y un $10 \%$ palizas (Pichardo, 2007). Por su parte, el $76.5 \%$ de los chicos y chicas de 15 a 29 años aceptaban el matrimonio homosexual y en torno al $65-80 \%$ aceptaban la legitimidad de la homosexualidad, frente al $20-30 \%$ que la rechazan (Injuve, 2008). Estudios recientes como el de Rodríguez-Castro, Lameiras, Carrera y Vallejo (2013) demuestran que los chicos son más homófobos que las chicas y, que ambos sienten más homofobia hacia los homosexuales de su mismo sexo. En esta misma línea se sitúa el estudio de Carrera, Lameiras, Rodríguez y Vallejo (2013) que afirman que son los chicos adolescentes quienes tienen actitudes más negativas hacia las personas gays y lesbianas y además también son los que presentan mayores niveles de sexismo. Otro estudio que da un paso más evaluando las actitudes hacia las personas que no conforman el género evidencia que siguen siendo los chicos los que muestran actitudes más discriminatorias tanto hacia las personas transexuales como hacia las personas homosexuales/lesbianas (Carrera, Lameiras, Rodríguez \& Vallejo, 2014). Estos datos ponen de manifiesto la existencia de actitudes homófobas en el colectivo adolescente del ámbito urbano, por lo que se hace necesario seguir profundizando en esta temática. Es por tanto, el objetivo de este estudio en analizar las actitudes hacia la diversidad sexual de los y las adolescentes del ámbito semi-rural de la provincia de Ourense.

\section{Método}

\section{Participantes}

La muestra está formada por 150 estudiantes de $2^{\circ}$ ciclo de Educación Secundaria de una localidad semi-rural de la provincia de Ourense (Galicia). De los cuales un $51.4 \%$ son chicos y $48.6 \%$ son chicas con una media de edad de 15.9 (DT = 1.07) y un rango que va de 14 a $\operatorname{los} 19$ años.

\section{Instrumentos}

Se ha aplicado un cuestionario ad hoc, que incluía datos sociodemográficos: género y edad, así como las siguientes escalas:

Escala de Homofobia Moderna de Raja y Stokes (1998) en la versión española de Rodríguez-Castro et al. (2013) que está formada por dos subescalas: la de actitudes hacia gays, con un total de 22 ítems; y la de actitudes hacia lesbianas, con un total de 24 ítems. Esta escala profundiza en tres dimensiones sutiles tanto hacia homosexuales como hacia lesbianas, que son: i) Malestar Personal que se define como la necesidad de evitar el contacto personal con los gays y las lesbianas, debido a una sensación de incomodidad cuando los gays y las lesbianas están presentes y/o la creencia de que los gays y las lesbianas están enfermos y son unos/as pervertidos/as; ii) Homofobia Institucional que hace referencia a las prácticas organizadas o sistemáticas de exclusión de las personas gays y lesbianas tanto a nivel gubernamental, empresarial, religiosa o profesional; y, iii) Desviación/ Cambiabilidad que está relacionada con la creencia de que las personas gays y lesbianas pueden cambiar su orientación sexual cuando ellos/as quieran. Con un formato de respuesta tipo Likert de 1 (muy en desacuerdo) a 5 (muy en desacuerdo) en la que a mayores puntuaciones interpretamos actitudes más positivas hacia la homosexualidad y el lesbianismo. El alpha de Cronbach de este estudio fue de .70 en la escala de homofobia y de .78 en la escala de Lesbofobia.

Escala de Sexismo Ambivalente (ASI) de Glick y Fiske (1996), en la versión española reducida de Rodríguez-Castro, Lameiras y Carrera (2009), con la que se miden las actitudes ambivalentes (hostiles y benevolentes) hacia las mujeres. La escala está compuesta por 12 ítems: seis evalúan el Sexismo Hostil (SH) y los otros seis miden el Sexismo Benevolente (SB) con un rango de respuesta tipo Likert que va de 0 (totalmente en desacuerdo) a 5 (totalmente de acuerdo), siendo las puntuaciones más altas las que representan el mayor nivel de sexismo. El $\alpha$ de Cronbach fue de .80 para el SH y de .70 para el SB.

\section{Procedimiento}

Seleccionados los centros de la localidad semi-rural de la provincia de Ourense (Galicia) nos pusimos en contacto por e-mail con la dirección de los centros explicando el objetivo del estudio y solicitando su colaboración. Aceptada la colaboración, finalmente se fijó fecha y hora para aplicar el cuestionario a los y las estudiantes. Los cuestionarios fueron aplicados en horario lectivo. Se contó con el consentimiento informado pasivo por parte del equipo de dirección del 
centro y de orientación. La aplicación de los cuestionarios trascurrió con normalidad con una duración de 25 minutos.

\section{Resultados}

Los resultados de este estudio evidencian que los chicos son más homófobos que las chicas (ver Tabla 1). Así, los chicos presentan actitudes más negativas hacia la homofobia institucional, hacia la desviación y el malestar personal frente a sus compañeras que tienen actitudes más positivas.

En cuanto a las subescalas de lesbofobia no se han detectado diferencias significativas entre chicos y chicas, excepto en la subescala de malestar personal, que son las chicas las que tienen un mayor malestar personal hacia las lesbianas (ver Tabla 1). Es importante destacar que los chicos muestran actitudes más positivas hacia el lesbianismo que hacia la homosexualidad.

En relación al nivel de sexismo de los y las adolescentes encontramos altas puntuaciones. Los chicos muestran un nivel alto de sexismo hostil frente a sus compañeras. Ambos por igual muestran el mismo nivel de sexismo benevolente, no detectándose diferencias significativas (ver Tabla 1).

Tal y como puede observarse en la Tabla 2, se presentan las correlaciones de las escalas de homofobia, lesbofobia con las de sexismo ambivalente (sexismo hostil y sexismo benevolente), constatamos que el sexismo hostil se relaciona negativamente con malestar personal, desviabilidad y homofobia institucional; también se relaciona con la lesbofobia institucional y la cambiabilidad. De forma que las personas más sexistas hostiles presentan actitudes más negativas hacia la homosexualidad y el lesbianismo.

Tabla 1.

Diferencias de medias en las subescalas de homofobia, lesbofobia y de sexismo ambivalente en función del género.

\begin{tabular}{cccc}
\hline & Género & $M(D T)$ & t-test \\
\hline Institucional_H & Chico & $2.24(.41)$ & $-3.77 * * *$ \\
& Chica & $2.56(.56)$ & \\
Malestar & Chico & $3.11(.81)$ & $6.55^{* * *}$ \\
Personal_H & Chica & $3.84(.42)$ & \\
Desviación_H & Chico & $1.43(.56)$ & $-4.22^{* * *}$ \\
& Chica & $2.05(1.10)$ & \\
Institucional_L & Chico & $3.12(.41)$ & -1.03 \\
& Chica & $3.02(.32)$ & \\
Malestar & Chico & $4.01(.71)$ & $-1.63 *$ \\
Personal_L & Chica & $3.83(.72)$ & \\
Desviación_L & Chico & $1.54(.90)$ & -.58 \\
& Chica & $1.33(.68)$ & \\
Sexismo Hostil & Chico & $4.22(1.24)$ & $-5.87 * * *$ \\
& Chica & $2.18(.81)$ & \\
S. Benevolente & Chico & $3.02(1.09)$ & -.120 \\
& Chica & $3.0(1.01)$ & \\
\hline
\end{tabular}

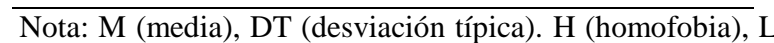
(lesbofobia); *p<.5; ***p<.001
En relación a la escala de sexismo benevolente solo se correlaciona fuertemente y de forma negativa con la subescala de malestar personal de Lesbofobia (ver Tabla 2).

Tabla 2.

Correlaciones entre las subescalas de homofobia $y$ lesbofobia con las del ASI.

\begin{tabular}{lc|c}
\hline & SH & SB \\
\hline Malestar Personal_Lesbianas & $-.42^{* *}$ & $-.60 * *$ \\
Institucional_Lesbianas & $-.39 * *$ & .06 \\
Desviación_Lesbianas & $-.46^{* *}$ & .01 \\
Malestar personal_Gays & -.08 & -.09 \\
Institucional_Gays & $.17 *$ & .03 \\
Desviación_Gays & $.19^{*}$ & -.04
\end{tabular}

Nota: SH (sexismo hostil), SB (sexismo benevolente). ${ }^{*} \mathrm{p}<.5$; **p.01

Para comprobar si el nivel de homofobia y de lesbofobia de los y las adolescentes del ámbito semi-rural es diferente del de los/as adolescentes del ámbito urbano se comparan las medias con las del estudio de Rodríguez-Castro et al. (2013) (ver Tabla 3). De forma general se puede observar que los chicos y las chicas del medio semi-rural muestran actitudes más negativas hacia la homosexualidad y el lesbianismo. Así podemos destacar las máximas diferencias en relación a la Desviación de Gays y la Desviación de Lesbianas, siendo los chicos y las chicas del ámbito semi-rural las que presentan unos altos niveles de discriminación hacia este colectivo.

Tabla 3

Estudio comparativo de medias en función de la localidad

\begin{tabular}{llcc}
\hline & género & $\begin{array}{c}\text { Estudio } \\
\text { urbano }^{I}\end{array}$ & $\begin{array}{c}\text { Estudio } \\
\text { semi-rural }^{-}\end{array}$ \\
\hline \multirow{2}{*}{ Malestar Personal_L } & Chica & 4.02 & 3.83 \\
& Chico & 3.87 & 4.01 \\
Institucional_L & Chica & 3.97 & 3.02 \\
& Chico & 3.61 & 3.12 \\
Desviación L & Chica & 4.61 & 1.33 \\
\multirow{3}{*}{ Malestar personal_Gays } & Chico & 4.20 & 1.54 \\
& Chica & 4.28 & 3.84 \\
Institucional_Gays & Chico & 3.25 & 3.11 \\
& Chica & 4.04 & 2.56 \\
Desviación_Gays & Chico & 3.42 & 2.24 \\
& Chica & 4.02 & 2.50 \\
\hline Nota: ${ }^{1}$ (Rodríguez-Castro & Chico & 3.79 & 1.43 \\
\hline
\end{tabular}

Nota: ${ }^{1}$ (Rodríguez-Castro et al., 2013); L (subescala Lesbofobia). 


\section{Discusión}

En relación a las actitudes homofóbicas hacia gays y lesbianas de los y las adolescentes del ámbito semi-rural, se constata en esta investigación que los chicos muestran más significativamente actitudes de homofobia hacia los gays que las chicas. Además, las puntuaciones significativamente diferentes que se han obtenido en las subescalas de homofobia y de lesbofobia, están en la línea de los principales estudios tanto en población adolescente (Injuve, 2008; Pichardo, 2007; Rodríguez-Castro et al., 2013; Carrera et al., 2013, 2014) como en población de estudiantes universitarios (Horn, 2007; Lingiardi, Falange, \& Augelli, 2005; Nuñez, Moreno \& Moral, 2011).

Es importante destacar que los chicos del ámbito semi-rural muestran actitudes más positivas hacia el lesbianismo que hacia la homosexualidad. Estos resultados están en la línea de estudios previos (Herek \& Carpintero, 1995; Raja \& Stokes, 1998). Una explicación a este hecho está relacionado con la cultura heteropatriarcal que erotiza las relaciones entre dos mujeres que son vistas como simples objetos sexuales a las que se les niega su placer sexual, es decir, son vistas como objetos sumisos del deseo masculino y se reafirma la construcción política de la heterosexualidad como principio organizador de las relaciones sexuales (Ruiz, 2006). De forma que la erótica lesbiana ha estado marcada a lo largo de la historia por estas creencias que la cultura masculina dominante ha cimentado y perpetuado, manteniendo así, el dominio de la sumisión y fetichismo de las relaciones sexuales entre dos mujeres (Lameiras et al., 2013).

Además, hemos también encontrado que las chicas del ámbito semi-rural tienden a expresar actitudes más positivas hacia el colectivo de homosexuales que hacia el de lesbianas. Esta tendencia también la evidencia el estudio Raja y Stokes (1998). De manera que nuestros resultados reflejan que las chicas del semi-rural tienden a creer, en mayor medida que los chicos, que las mujeres lesbianas son pervertidas y suelen estar más cómodas con la presencia de una pareja de gays que con la de una pareja de lesbianas.

Otro resultado interesante es que los chicos y chicas con actitudes más discriminatorias hacia las minorías sexuales también muestran mayores actitudes sexistas hostiles hacia las mujeres. En la línea del estudio de Carrera et al. (2013) que evidenciaba que a mayor sexismo tradicional hacia la mujer se da un mayor nivel de homofobia, de lesbofobia y de transfobia en los y las adolescentes.

Al comparar los niveles de homofobia y de lesbofobia entre los y las adolescentes del ámbito urbano (Rodríguez-Castro et al., 2013) con los de nuestro estudio encontramos que de forma general, los chicos y las chicas del medio semi-rural muestran actitudes mucho más negativas hacia la homosexualidad y el lesbianismo que los del ámbito urbano. Así podemos destacar que las mayores diferencias, se detectan en las subescalas de Desviación de Gays y la de Desviación de Lesbianas. Así, los chicos y las chicas del ámbito semi-rural tienden a evitar el contacto personal tanto con los homosexuales como con las lesbianas, debido a que los consideran unos depravados. Estos datos ponen de manifiesto lo importante que es trabajar desde el sistema educativo con este colectivo de adolescentes, en pro de la aceptación de la diversidad sexual. Para lograr un cambio positivo en las actitudes hacia la diversidad sexual de los y las adolescentes, específicamente los del ámbito semi-rural, los estudios evidencian que las personas que conocen a otras personas LGTBI presentan actitudes más positivas hacia ellas; es decir, el hecho tener apertura mental y estar en contacto con otros colectivos, se considera uno de los factores que más influye positivamente sobre las actitudes de las personas (Eurobarómetro, 2008). Por lo tanto, los resultados evidencian la necesidad urgente de seguir trabajando en los centros educativos desde una perspectiva coeducativa que se base en el respeto, la tolerancia, la igualdad y la diversidad sexual.

\section{Referencias}

Aosved, A. C. \& Long, P. J. (2006). Co-occurrence of Rape Myth Acceptance, Sexism, Racism, Homophobia, Ageism, Classism, and Religious Intolerance. Sex Roles, 55, 481-492.

Carrera, M. V., Lameiras, M., Rodríguez, Y. \& Vallejo, P. (2013). Bullying among Spanish Secondary Education Students: The role of Gender Traits, Sexism, and Homophobia. Journal of Interpersonal Violence, 28(14), 2915-2940. doi: 10.1177/0886260513488695

Carrera, M.V., Lameiras, M., Rodríguez, Y. \& Vallejo, P (2014). Spanish adolescents' attitudes toward transpeople: proposal and validation of a short form of the Genderism and Transphobia Scale. Journal of Sex Research, 51(6), 654-666. doi: http://dx.doi.org/10.1080/00224499.2013.773577

Consejo Europeo. (2011). Discrimination on Grounds of sexual Orientation and gender Identity in Europe. Recuperado

de http://www.coe.int/t/Commissioner/Source/LGBT/LG BTStudy2011_en.pdf

España, A., Guerrero, R., Farré, J. M., Canella, J. \& Abós, R. (2001). La homofobia en el medio universitario. Un estudio empírico. Cuadernos de Medicina Psicosomática y Psiquiatría de Enlace, 59, 41-55.

Eurobarómetro (2008). Discrimination in the European Union: Perceptions, Experiences and Attitudes. European Commission, Special Eurobarometer, 296.

Glick, P. \& Fiske, S. T. (1996). The Ambivalent Sexism lnventory: Differentiating hostile and benevolen sexism. Journal of Personality and Social Psychology, $70,491-512$

Guash, O. (2006). Héroes, Científicos, Heterosexuales y Gays. Los varones en la perspectiva de género. Barcelona: Bellaterra.

Herek, G. M. \& Carpintero, J. P. (1995). Black heterosexuals' attitudes toward lesbian and gay men in United Status. Journal of Sex Research, 32, 95-105.

Horn, S. S. (2007). Adolescents' Acceptance of Same-Sex Peers Based on Sexual Orientation and 
Gender Expression. Journal of Youth and Adolescence, 36, 363-371.

Injuve (2008). Informe juventud en España 2008. Madrid: autor.

International Lesbian, Gay, Bisexual, Trans \& Intersex Association (ILGA). (2017). The human rights situation for LGBTI people - country-by-country. Recuperado de https://www.ilga-europe.org/

Katz, I., Wackenhut, J. y Hass, R. G. (1986). Racial ambivalence, value duality, and behavior. En J. F. Dovidio \& S. L. Gaertner (Eds.), Prejudice, discrimination, and racism (pp. 35-59). Nueva York: Academic Press.

Lameiras, M., Carrera, M. V. \& Rodríguez, Y. (2013). Sexualidad y Salud. El estudio de la sexualidad humana desde la perspectiva de género. Vigo: Servicio Publicaciones Universidad de Vigo.

Lingiardi, V., Falange, S. \& Augelli, R. D. (2005). The evaluation of Homophobia in an Italian Sample. Archives of Sexual Behaviour, 34, 81-93.

Martín Romero, L. (2008). Sondeo COGAM. Homofobia 2008. COGAM. Recuperado de http://www.cogam.es/secciones/stop-homo-transfobia/ documentos/i/429153/492/resultados-del-sondeo-de-s os-homofobia-2008

McConahay, J. B. \& Hough, J. C. Jr. (1986). Symbolic racism. Journal of Social Issues, 32, 23-45.

Nuñez, M., Moreno, M. P. \& Moral, F. (2011). Modelo causal del prejuicio religioso. Anales de Psicología, 27, 852-861.

Pettigrew, T. F. (1989). The nature of modern racism in the United States. Revue International de Psychologie Sociale, 2, 291-303.

Pichardo, J. I. (2007). Actitudes ante la diversidad sexual de la población adolescente de Coslada (Madrid) y San Bartolomé de Tirajana (Gran Canaria). Madrid: FELTGB, COGAM.

Quiles, N., Betancor, V., Rodríguez, R., Rodríguez, A. \& Coello, E. (2003). La medida de la homofobia manifiesta y sutil. Psicothema, 15, 197-204.

Raja, S. H. \& Stokes, J. P. (1998). Assessing Attitudes toward lesbians and Gay men: The modern Homophobia Scale. Journal of Gay, Lesbian and Bisexual Identity, 3, 113-134.

Rodríguez-Castro, Y., Lameiras, M. \& Carrera, M.V. (2009). Validación de la versión reducida de las escalas ASI y AMI en una muestra de estudiantes españoles. Psicogente, 12 (22), 284-295.

Rodríguez-Castro, Y., Lameiras, M., Carrera, M. V. \& Vallejo, P. (2013). Validación de la Escala de Homofobia Moderna en una muestra de adolescentes. Anales de Psicología 29 (2), 523-533. doi: http://dx.doi.org/10.6018/analesps.29.2.137931

Ruiz, P. R. (2006). Los modos de representación de la sexualidad lesbiana: coexistiendo con la heteronormatividad. En Bosch, E., Ferrer, V. \& Navarro, C. (Eds.), Los feminismos como herramientas de cambio social (pp. 359-369). Palma: Edició Universitat de les Illes Balears.

Swim, J. K., Aikin, W. S., HaII, W. S. \& Hunter, B. A. (1995). Sexisn, and Racism: Old-fashioned and
Modern Prejudices. Journal of Personality and Social Psychology, 68, 199-214.

Tougas, F., Brown, R., Beaton, A. M. \& Joly, S. (1995). Neosexism: Plus Ca Change, Plus C`est Pareil. Journal of Personality and Social Psychology, 21, 842-849.

Viñuales, O. (2002). Lesbofobia. Barcelona: Bellaterra. 